\title{
Über die Löslichkeit des Graphits in geschmolzenem Eisen.
}

\author{
Von Rudolf Ruer und Jubios Braen.
}

Mit 5 Figuren and 3 Tabellen im Text.

Utber den Verlauf der Schmelzkurven der übereutektischen EisenKohlenstofflegierungen gehen die Ansichten noch weit auseinander. ${ }^{1}$ )

Die im nachfolgenden mitgeteilten Versuche bezweckten eine möglichst einwandfreie Bestimmung der Löslichkeit des Graphits in geschmolzenem Eisen bis hinauf zu hohen Temperaturen. Es sollte-dabei in der Weise verfahren werden, daß geschmolzenes Eisen mit überschüssigem Graphit bis zur Sättigung erhitzt und dann so schnell abgekühlt wurde, daß eine Wiederausscheidung des gelösten Graphits als elementarer Kollenstoff nicht stattfand. Diese Absicht kcnnte nur bis zu etwa $1500^{\circ} \mathrm{C}$ verwirklicht werden, doch wiesen die erhaltenen Resultate den Weg, die Bestimmungen auch bei höheren Temperaturen durchzuführen.

Das zu den Versuchen benutzte Eisen war schwedisches Holzkohlenroheisen, welches $3,8 \% \mathrm{C}, 0,06 \% \mathrm{Si}$, kein Mangan, $0,02 \% \mathrm{~S}$ und $0,06 \% \mathrm{P}$ enthielt. Der benutzte Graphit war von der Firma E. MERck, Darmstadt, bezogen und hinterlieB 0,18\% Glührückstand.

Die 'Temperaturmessung geschah bis $1700^{\circ} \mathrm{C}$ auf thermoelettrischem Wege, bei höheren Temperaturen mit Hilfe eines optischen Pyrometers von Holbons und Kurlbaum, welches Herr Geheimrat Prof. Dr. A. Classen freundlichst zur Verfügung stellte. Fss sei ihm auch an dieser Stelle bestens dafür gedankt.

Die Abschreckung der Schmelze erfolgt am zweckmäßigsten nach der von Hanemann $^{2}$ ) angegebenen Methode, die Schmelze in eine ganz enge Metallkokille einzugießen. Die zu den im nachfolgenden beschriebenen Versuchen benutzte, durch Fig. 1 dargestellte Kokille bestand aus Kupfer. Die Dicke der Einlageplatte nahm von $1 \mathrm{~mm}$ bis zu $0,4 \mathrm{~mm}$ von oben nach unten hin ab. Die erhaltenen GuBplatten hatten eine Dicke von $0,5-0,8 \mathrm{~mm}$.

1) H. HanemanN, Stahl und Eisen 31 (1911), 333; Z. anorg. Chem. 84 (1913), 1; O. RuFF und O. Goeckr, Metallurgie 8 (1911), 417; O. RUFF, Metallurgie 8 (1911), 456, 497; Z. anorg. Chem. 89 (1914), 39; O. RuFF und W. Bormann, Z. anorg. Chem. 88 (1914.) 397; N. M, WrTTORF, Z. anorg. Chem. 79 (1912), 1.

2) H. HANEMANN Stuhl und Eisen 31 (1911), 333. 
Noch weiter mit der Dicke der Platte herunterzugehen, erwies siọh als unmöglich, weil dann die Menge der in die Kokille einflieBenden Schmelze zu gering wurde.

Zum Zwecke der Analyse wurden die Proben im Diamantmörser so weit gepulvert, daB alles durch ein Metallsieb von 0,5 mm Maschenweite hindurchging. Die Bestimmung des Gesamtkohlenstoffs geschah durch Verbrennung im Marsofen. Die Graphitbestimmung erfolgte in der üblichen Weise durch BehandlungmitSalpetersäure von 1,18 spezifischem Gewichte, zuletzt auf dem Wasserbade, bis zur Lösung des Eisens, Filtration des verbleibenden Rückstandes durch ein Asbestfilter und Verbrennung desselben im Marsofen nach vorheriger Trocknung bei $100^{\circ} \mathrm{C}$. $\mathrm{Da}$

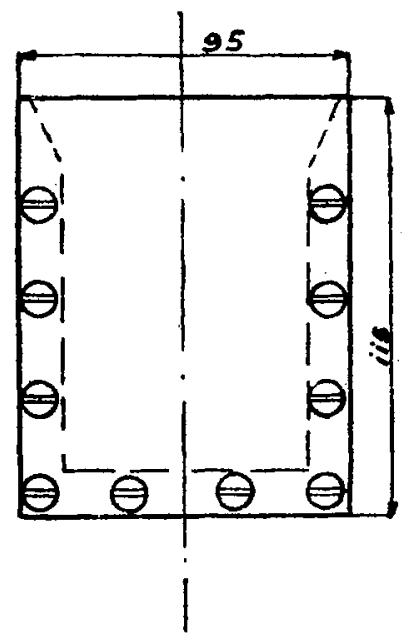

Fig. 1. bei reinem Elektrolyteisen nach dieser Methode ein Gehalt von $0,03 \%$ Graphit gefunden wurde, so wurden von den gefundenen Graphitgehalten $0,08 \%$ abgezogen. Der chemisch gebundene Kohlenstoff ergibt sich aus der Differenz zwischen dem Gesamtkohlenstoff- und dom Graphitgehalt.

Bei Ausführung der Untersuchung standen Mittel aus der Monitz Honigmann-Stiftung zur Verfügung.

\section{Thermoelektrische Temperaturbestimmung.}

Die Resultate der Versuche, bei denen die Temperatur auf thermoelektrischem Wege gemessen wurde, sind in Tabelle 1 zusammengestellt und in Fig. 2 wiedergegeben.

Als Schmelzgefäße dienten bei den Versuchen 1-10 Probierröhren aus Porzellan von $15 \mathrm{~mm}$ lichtem Durchmesser und $100 \mathrm{~mm}$ Höhe, bei Versuch 11 eine solche aus Magnesia, welche von der Porzellanmanufaktur, Berlin, bezogen war, bei Versuch 12 eine solche aus Kohle, von der Firma C. Conradty, Nürnberg, geliefert. In diesen wurden mit Hilfe eines TAMMANs schen Kohlerohr-KurzschluBofens etwa $20 \mathrm{~g}$ des schwedischen Holzkohlenroheisens mit so viel 
Tabelle 1.

\begin{tabular}{r|c|c|c|c}
\hline Nr. & $\begin{array}{c}\text { Temperatur } \\
\text { Grad C }\end{array}$ & $\begin{array}{c}\text { Gesamt- } \\
\text { C-Gehalt } \%\end{array}$ & $\begin{array}{c}\text { Graphitgehalt } \\
\%\end{array}$ & $\begin{array}{c}\text { Gebundener } \\
\text { Koblenstoff \% }\end{array}$ \\
\hline 1 & 1190 & 4,34 & 0 & 4,34 \\
2 & 1215 & 4,41 & 0 & 4,41 \\
3 & 1265 & 4,51 & 0,03 & 4,48 \\
4 & 1305 & 4,64 & 0,04 & 4,60 \\
5 & 1385 & 4,85 & 0,06 & 4,79 \\
6 & 1420 & 4,96 & 0,04 & 4,92 \\
7 & 1480 & 5,04 & 0,08 & 4,96 \\
8 & 1530 & 5,22 & 0,22 & 5,00 \\
9 & 1580 & 5,32 & 0,29 & 5,03 \\
10 & 1615 & 5,35 & 0,55 & 4,80 \\
11 & 1670 & 5,51 & 1,36 & 4,15 \\
12 & 1710 & 5,72 & 1,40 & 4,32
\end{tabular}

Graphit zusammengeschmolzen, daß nach erfolgtem Ausgießen in die Kokille noch etwa 0,1-0,2 g Graphit im Röhrchen zurückblieben.

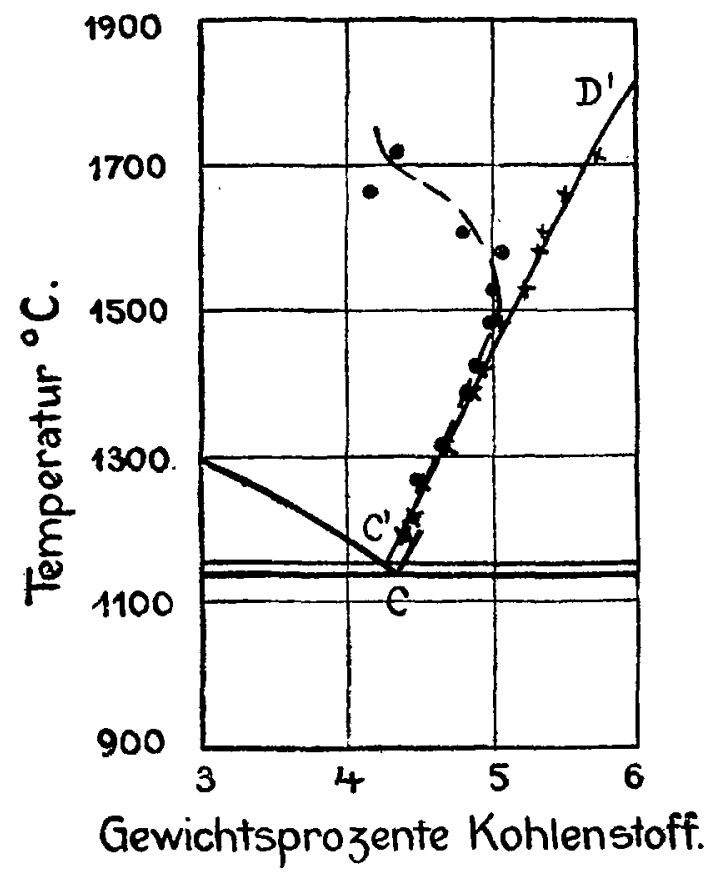

Fig. 2.

Die Probierröhren waren in der üblichen Weise durch ein zweifach durchbohrtes Messinghütchen verschlossen. Zwecks Verhütung des Luftzutritts wurde während der Schmelzung ein lebhafter Strom von Stickstoff durchgeleitet, welcher zunä.chst eine mit alkalischer 
Pyrogallollösung, sodann eine mit konzentrierter Schwefelsäure gefüllte Waschflasche und schließlich eine mit erhitzten Kupferspänen gefüllte Röhre passierte. Die Schmelzen wurden langsam auf die in Spalte 2 der Tabelle 1 angegebene Temperatur erhitzt und diese etwa eine Viertelstunde lang bei häufigem Umrühren konstant gehalten. Dann wurde bei konstant gehaltenem Heizstrom das Thermoelement aus der Schmelze entfernt, diese einen Augenblick absitzen gelassen und durch Eingießen in die Kokille abgeschreckt.

Die Temperaturmessung geschah durch ein. Thermoelement aus Platin-Platinrhodium, das durch Einschluß in ein unten zugeschmolzenes Quarzrohr vor Berührung mit der Schmelze geschützt war. Zur Eichung des Thermoelementes dienten die Schmelzpunkte von Kupfer $\left.{ }^{1}\right)\left(1084^{0} \mathrm{C}\right)$, Nickel $\left.{ }^{2}\right)\left(1451^{\circ} \mathrm{C}\right)$, Eisen $\left.{ }^{3}\right)\left(1528^{\circ} \mathrm{C}\right)$ and Platin $\left.{ }^{4}\right)$ $\left(1764^{\circ} \mathrm{C}\right)$ als Fixpunkte. Die Eichung nach dem Schmelzpunkte des Platins kann, da die Platinrhodiumlegierung einen höheren Schmelzpunkt hat als das reine Platin, analog der Drahtmethode durch Durchschmelzen des Thermoelementes erfolgen, wobei in folgender Weise verfahren wurde. Das Thermoelement, dessen einer Draht durch eine dünne Quarzkapillare hindurchgezogen war, befand sich in einem unten zugeschmolzenen $130 \mathrm{~mm}$ langen Quarzrohr von $5 \mathrm{~mm}$ innerem Durchmesser und $2 \mathrm{~mm}$ Wandstärke, welches selbst wieder in ein Reagenzrohr aus Magnesia eingestellt wurde. In das durch ein zweifach durchbohrtes Messinghütchen verschlossene Magnesiarohr wurde während der Erhitzung ein rascher Strom von Stickstoff eingeleitet. Auf diese Weise gelang es, den Zutritt von Kohlenoxyd zum Thermoelement zu verhindern. Die Durchschmelzung des Thermoelementes erfolgte bei 18,6 Millivolt, wenn die Temperatur der kalten Lötstelle $0^{\circ} \mathrm{C}$ betrug. Da das zur Temperaturmessung dienende von der Firma Sirmens \& Halske gelieferte Millivoltmeter nur bis 18 Millivolt rejchte, so wurde die Skala darüber hinaus auf der Glasdeckscheibe fortgeführt.

Die in Tabelle 1, Spalte 3-5 angegebene Zusammensetzung der abgeschreckten Schmelzen läßt erkennen, daß der Gehalt an Graphit bis über $1200^{\circ} \mathrm{C}$ Null ist und auch bis $1500^{\circ} \mathrm{C}$ noch unter $0,1 \%$ beträgt. Oberhalb $1500^{\circ} \mathrm{C}$ nimmt er schnell zu und beträgt bei $1600^{\circ} \mathrm{C}$ schon $0,5 \%$. In Fig. 2 stellt die ausgezogene Kurve $C^{\prime} D^{\prime}$

1) Holborn und DAy, Drudes Ann. 2 (1900), 545.

2) R. Ruer, Z. anorg. Chem. 51 (1906), 225.

3) R. RUER und R. KLesper, Ferrum 11 (1914), 257.

4) Physikalisch-Technische Reichsanstalt, Ann. d. Phys. IV 48 (1915), 1034. 
UNIV BIBL BERLIN

den Gesamtkohlenstoffgehalt in Abhängigkeit von der Temperatur dar, wobei die Resultate der einzelnen Versuche durch Kreuze gekennzeichnet sind, die von $C^{\prime}$ ausgehende gestrichelte Kurve dagegen den Gehalt an gebundenem Kohlenstoff, wobei die Resultate der einzelnen Versuche durch Punkte wiedergegeben sind. Man erkennt, daß die beiden Kurven in ihrem ersten Teil vollkommen zusammenfallen und daB sie noch bei $1500^{\circ} \mathrm{C}$ sehr wenig voneinander abweichen. Da von $1500^{\circ} \mathrm{C}$ an der Graphitgehalt sehr schnell zunimmt, so tritt mit höherer Temperatur sogar eine Verminderung an gelöstem Kohlenstoff ein, was durch das Zurückbiegen der gestrichelten Kurve zum Ausdruck kommt. Die Ursache für diese starke Zunahme des Graphitgehalts bei steigender Temperatur ist ohne Frage darin zu suchen, daß für die kohlenstoffreicheren Reguli die Abschreckung nicht genügend schroff war und daß die infolgedessen in gröBerer Anzahl entstandenen Graphitkeime eine impfende Wirkung ausübten. Das ergibt sich auch daraus, da $B$ bei den ersten Versuchen, bei denen zum Abschrecken nicht die oben beschriebene, sondern eine Kokille benutzt wurde, welche Gußplatten von etwa $1,8 \mathrm{~mm}$, also etwa dreifacher Dicke lieferte, die starke Zunahme des Graphitgehaltes mich sohon bei tieferer Temperatur, rund $1400^{\circ} \mathrm{C}$, bemerkbar machte.

Bis $1500^{\circ} \mathrm{C}$ zeigen Gesamtkohlenstoffgehalt und Gehalt an gebundenem Kohlenstoff eine so weitgehende Übereinstimmung, daB, wenn man den Gehalt an gebundenem Kohlenstoff als Löslichkeit des Graphits ansieht, der durch ungenügendes Abschrecken verursachte Fehler weniger als $0,1 \%$ beträgt. Die Versuche ergeben also, daß von dem mit überschüssigem Graphit erhitzten Eisen bis $1200^{\circ} \mathrm{C}$ kein Graphit in Suspension gehalten wurde und daB es bei $1500^{\circ} \mathrm{C}$ jedenfalls weniger als $0,1 \%$ war. Daraus ist zu schließen, $\mathrm{da} B$ geschmolzenes Eisen unter normalen Verhältnissen überhaupt keine in Betracht kommende Graphitmenge in Suspension zu halten vermag und daß bei den unter- und oberhalb $1500^{\circ} \mathrm{C}$ ausgeführten Versuchen der Gesamtkohlenstoffgehalt die Löslichkeit des Graphits im Eisen wiedergibt, während dem Gehalt an gebundenem Kohlenstoff, als von einem Nebenumstand, der Abschreckungsgeschwindigkeit abhängig, keine Bedeutung beizumessen ist.

Man könnte gegen die Versuche den Einwand erheben, daß die Schmelze nicht mit Graphit gesättigt gewesen wäre. Dies ist schon aus dem Grunde unwahrscheinlich, weil bei der niedrigsten Temperatur $\left(1190^{\circ} \mathrm{C}\right.$, Versuch 1) bereits ein etwas übereutektischer Kohlenstoffgehalt gefunden wurde. Es wurden jedoch noch zwei Versuche 
ansgeführt, bei denen das Gleichgewicht von höherer Temperatur aus erreicht wurde. Die Schmelzen wurden mit überschüssigem Graphit auf etwa $1550^{\circ} \mathrm{C}$ erhitzt, darauf langsam auf $1270^{\circ} \mathrm{C}$ abkühlen gelassen und dann abgeschreckt. Der Gesamtkohlenstoffgehalt der abgeschreckten Proben betrug das eine Mal 4,57\% bei 0,02\% Graphitgehalt, das andere Mal 4,55\% bei 0,03\% Graphitgehalt, also innerhalb der Grenzen der Versuchsfehler ebensoviel, als wenn das Gleichgewicht von niederer Temperatur aus erreicht wurde, wie, dies bei sämtlichen in Tabelle 1 mitgeteilten Versuchen der Fall war.

Weiterhin könnte man einwenden, daB beim AusgieBen der Sehmelzen in die Kokille eine Oxydation und dadurch ein Abbrand des anfgenommenen Kohlenstoffs stattfände. Daß dies nicht der Fall ist, ergibt sich daraus, daB Schmelzen, welche unter weiterem Einleiten von Stickstoff im Probierrohre außerhalb des Ofens erkalteten, den gleichen Gehalt an Gesamtkohlenstoff aufwiesen, als die unter sonst gleichen Umständen abgeschreckten Schmelzen.

Die Extrapolation der Lỏslichkeit des Graphits auf niedere Temperatur liefert für $1152^{\circ} \mathrm{C}$, die Kristallisationstemperatur des Graphiteutektikums ${ }^{1}$ ), den Wert $4,25 \% \mathrm{C}$. Demgemäß ergibt sich für den Kohlenstoffgehalt des Zementitentektikums, welcher den des Graphiteutektikums um

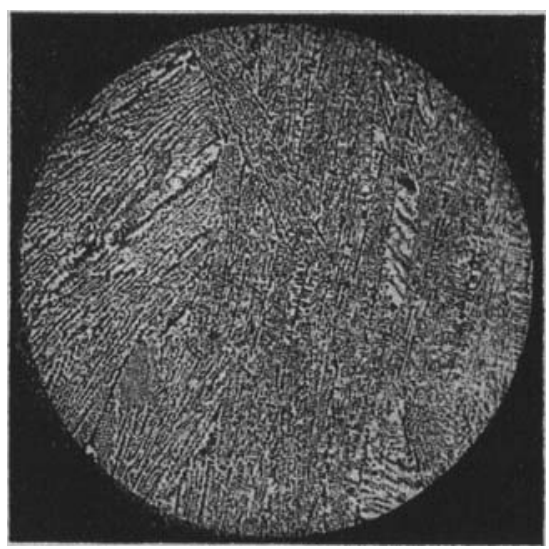

Fig. 3. Eisenkohlenstofflegierung mit $4,34 \% \mathrm{C}$, abgeschreckt. Mit glkoholischer Pikrinsüure geătzt. 280 fache VergrōBerung. $0,05 \%$ übertrifft $\left.{ }^{2}\right), 4,30 \%$ C.

Das ist ein etwas höherer Wert, als man bisher gewöhnlich annahm $(4,20 \% \mathrm{C})$.

In der Tat zeigte die bei $1190^{\circ} \mathrm{C}$ abgeschreckte Probe, in welcher $4,34 \%$ Kohlenstoff gefunden wurde (Tabelle 1, Versuch 1 ), ein rein entektisches Gefüge und enthielt weder Tannenbaumkristalle noch Zementitnadeln (Fig. 3). Man kann also auf sehr bequeme Weise

1) R. Rome und F. Gorrans, Ferrum 14 (1917), 170.

2) R. Ruge und F. Gohrens, Ferrum 14 (1917), 176. 
reines Zementiteutektikum in der Weise herstellen, daB man ein. etwas untereutektisches Roheisen mit überschüssigem Graphit oder auch im Kohletiegel bei möglichst niederer. $1200^{\circ} \mathrm{C}$ nicht übersteigender Temperatur zum Schmelzen bringt und dann vollkommen abschreckt.

Schließlich sei noch bemerkt, daß die Aufnahme der Abkühlungskurve eines mit überschüssigem Graphit erhitzten Eisens zwischen $1720^{\circ} \mathrm{C}$ und seiner eutektischen Erstarrungstemperatur keine Wärmetönung erkennen ließ. Daraus ergibt sich, da $\beta$ in diesem Temperaturintervall kein Stabilitätswechsel der primär ausgeschiedenen Kristallart stattfindet, wenigstens keiner, der mit merkbarer Wärmetönung verknüpft ist.

\section{Optische Temperaturbestimmung.}

Das zu der optischen Temperaturbestimmung benutze Pyrometer von Holborn und Kurlbaum war von der Physikalisch-Technischen Reichsanstalt gemäß ihrer Bekanntmachung vom Dezember 1915') geeicht. Die durch diese Bekanntmachung definierte Temperaturskala stimmt mit der der Eichung des benutzten Thermoelementes zugrunde gelegten Skala überein.

Da die Probierrohre aus Magnesia schon bei etwa $1800^{\circ} \mathrm{C}$ stark zu rauchen anfingen, wodurch die Genauigkeit der Temperaturmessung beeinträchtigt wurde, so wurden als Schmelzgefäße die schon oben erwähnten, von der Firma C. Conkadty, Nürnberg, gelieferten Probierröhren aus Kohle verwandt. Sie hatten einen Durchmesser von $15 \mathrm{~mm}$, eine Wandstärke von $2,5 \mathrm{~mm}$ und eine Länge von $100 \mathrm{~mm}$. Auch diese begannen schon unterhalb $2000^{\circ} \mathrm{O}$ einen Rauch abzugeben, der sich an den oberen, kälter gebliebenen Teilen als weißer Beschlag absetzte. Die Rohre wurden daher vor ihrer Verwendung zunächst einige Tage in kalte verdünnte Flußsäure eingesetzt, darauf mit Wasser abgespült und mit verdünnter Salpetersäure und zuletzt mit destilliertem Wasser ausgekocht. Nachdem sie dann in der Hitze getrocknet waren, wurden sie bei hoher, $2000^{\circ} \mathrm{C}$ wesentlich ühersteigender l'emperatur so lange geglüht, bis die Rauchentwicklung fast ganz aufgehört hatte. Die auf diese Weise vorbereiteten Probierrohre ließen sich meist bis etwa $2400^{\circ} \mathrm{C}$ verwenden, ohn $\Theta$ da $B$ die Genauigkeit der Temperaturmessung durch Rauchentwicklung wesentlich beeinträchtigt wurde.

^) Physikalisch-Technische Reichsanstalt, Ann. d. Phys. IV 48 (1915), 1034. 
Das Probierrohr war durch ein dreifach durchbohrtes Hütchen verschlossen. Durch die mittlere Öffnung wurde mittels des Pyrometerfernrohres die Oberfläche der Schmelze anvisiert, während von den beiden seitlichen Öffnungen die eine zur Aufnahme des $\mathrm{Zu}$ leitungsrohres für das als schützende Atmosphäre benutzte Gas, die andere zur Aufnahme eines als Rührer verwendeten Kohlestabes diente.

Eine gleichzeitige Temperaturbestimmung auf optischem und thermoelektrischem Wege, wobei das Schutzrohr des Thermoelementes durch die für den Kohlestab bestimmte Öffnung eingeführt wurde, ergab zwischen den Temperaturen 1200 und $1500^{\circ} \mathrm{C}$ eine gute Übereinstimmung der mit beiden Verfahren ermittelten Werte, indem die Differenzen in den meisten Fällen nicht mehr als $\pm 10^{\circ} \mathrm{C}$ betrugen. Das leere, durch ein Messinghütchen verschlossene Kohlerohr stellt daher einen schwarzen Körper dar. Wurde im Kohlerohr Eisen geschmolzen, so blieb die Übereinstimmung zwischen optischer und thermoelektrischer Temperaturmessung nur dann eine ebenso gute, wenn die Menge des Eisens nicht mehr als etwa $10 \mathrm{~g}$ betrug. Nahm man dagegen $20 \mathrm{~g}$, so ergab die optische Messung zu niedrige Werte. Es rührt dies anscheinend daher, daß die Lötstelle des Thermoelementes sich am Boden des Röhrchens befand, wo die Temperatur höher war, als an der Oberfläche der Schmelze. Daher wurden zu den Versuchen nur Mengen von je $10 \mathrm{~g}$ Eisen verwandt.

Zwei bei $1558^{\circ}$ und $1312^{\circ} \mathrm{C}$ angestellte Versuche, bei denen die Temperatur thermoelektrisch gemessen wurde, ergaben, daß das Eisen die gleiche Menge Kohlenstoff aufnimmt, gleichgültig, ob man als Schmelzgefäße Probierröhren aus Porzellan oder aus Kohle verwendet. Weiterhin war es bei Verwendung von Kohleröhren ohne Einfluß auf das Resultat, wenn ein Graphitzusatz unterblieb. Auch konnte man bei den über $1800^{\circ} \mathrm{C}$ ausgeführten Schmelzen beobachten, daß die innere Wandung des Kohlerohres, dort wo sie mit dem Eisen in Berührung gewesen war, mit Graphitblättchen bedeckt war. Unter diesen Umständen wurde bei den im folgenden mitgeteilten Versuchen von einem Graphitzusatz abgesehen. Es geschah dies hauptsächlich aus dem Grunde, weil das mit Kohlenstoff gesättigte Eisen bei ganz hohen Temperaturen sehr zähflüssig wird und dann immerhin mit der Möglichkeit zu rechnen ist, daß durch das Rühren in das Innere der Schmelze gelangte feine Graphitteile sich nicht von ihr abtrennen können. Diese Gefahr besteht natürlich in viel geringerem Maße, wenn das Eisen nur mit der glatten Oberfläche der Gefäßwand und des Rührers in Berührung kommt. 
Um in dem Tammannschen Kohlerohr-KurzschluBofen Temperaturen über $2000^{\circ} \mathrm{C}$ zu erzeugen, ohne die Außenteile des Ofens allzu stark zu erhitzen, erwies es sich als zweckmäßig, den mittleren Teil des Heizrohres auf eine Wandstärke von etwa $2 \mathrm{~mm}$ abzudrehen (Fig. 4). Es konnte so mit einer Stromstärke von $350 \mathrm{Amp}$. eine

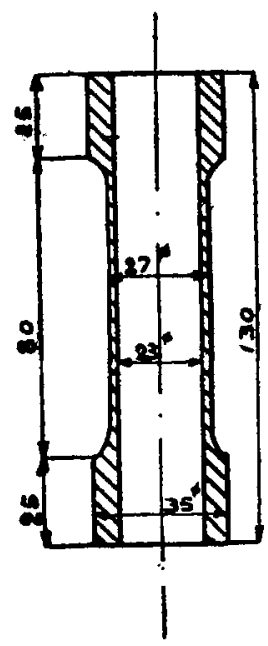

Fig. 4. Temperatur von etwa $2700^{\circ} \mathrm{C}$ erreicht werden. Allerdings dürfen die abgedrehten Rohre nicht allzu fest eingespannt werden, ( da sie sonst beim Erkalten des Ofens leicht reißen.

Als schützende Atmosphäre wurden Stickstoff, Wasserstoff, Kohlenoxyd, Rohargon und Reinargon verwandt. Das Rohargon war von der Gesellschaft für Lindes Eismaschinen, Abteilung: Gasverflüssigung, Höllriegelskreuth bei München bezogen und enthielt 88,3\% Argon, 9,7\% Stickstoff und $2 \%$ Sauerstoff. Es wurde vor der Verwendung in derselben Weise wie der Stickstoff (s. o.) getrocknet und von Sauerstoff befreit. Zur Herstellung des Reinargons wurde das Rohargon zwecks Entfernung des Stickstoffa so oft über glühende Calciumspäne geleitet, bis eine wesentliche Nitridbildung nicht mehr $\mathrm{zu}$ beobachten war. Der Grund, aus dem außer Stickstoff auch moch Wasserstoff und Kohlenoxyd als schützende Atmosphäre benutzt wurden, war der, den Einwand auszuschließen, daB der Stickstoff bei sehr hohen Temperaturen nicht als indifferentes Gas angesehen werden könne. Gegen die Berechtigung dieses Einwandes spricht allerdings schon der Umstand, daB die erkalteten Proben nur einen ganz belanglosen Stickstoffgehalt, durchschnittlich $0,007 \%$, anfwiesen und $d a ß$ sie keine schaumige, auf eine Gasentwicklung beim Erkalten hindeutende Beschaffenheit zeigten. Die Verwendung von Argon erfolgte, weil nach RuFF ${ }^{1}$ ) dieses Gas, welches im Gegensatze zu den oben genannten bei keiner Temperatur mit Kohlenstoff reagiert, keine Veranlassung tzum Auftreten von Rußwolken gibt. Dieser Vorzug des Argons kam bei den benutzten Probierröhren aus Kohle nicht ganz zur Geltung, da deren zufällige Beschaffenheit, je nachdem sie mehr oder minder gut ausgewaschen and ausgeglüht waren, einen größeren Einfluß auf die Rauchbildung

1) Row, Z. Elektrochem. 24 (1918), 157-162. 
auszuüben schien, als die Art des verwendeten Schutzgases. Immerhin hatte es den Ansebein, als ob bei Verwendung von Wasserstoff die Rauchbildung etwas früher auftrat, als bei den anderen Gasen.

Das Abschrecken der Schmelze durch Ausgieben in die Kokille war bereits bei $2000^{\circ} \mathrm{C}$ durch die Zähflüssigkeit der Sohmelze erschwert. Nachdem durch zwei bei $2000^{\circ} \mathrm{C}$ ausgeführte Versuche nochmals festgestellt war, daB es auf den Gesamtkohlenstoffgehalt der erkalteten Proben keinen EinfluB ausübte, wenn die Schmelzen, anstatt in die Kokille ausgegossen zu werden, unter weiterem Einleiten von Stickstoff im Probierrohre außerhalb des Ofens erkalteten, wurde das letztere Verfahren als das bequemere gewählt. Die Schmelzen wurden daher im Kohleprobierrohre unter Einleiten eines lebhaften Gasstromes bei häufigem Umrühren mittels des Kohlestabes besonders zuletzt sehr langsam auf die Versuchstemperatur erhitzt, der Kohlestab aus der Schmelze entfernt, das Probierrohr sus dem Ofen herausgenommen und erkalten gelassen.

Tabelle 2.

\begin{tabular}{|c|c|c|c|c|c|c|c|c|}
\hline \multirow{2}{*}{$\begin{array}{c}\text { Tempe- } \\
\text { ratur }{ }^{\circ} \mathrm{C}\end{array}$} & \multicolumn{8}{|c|}{ Gesamtkohlengtoffgehalt in Gewichtsprozenten } \\
\hline & $\begin{array}{l}\text { Stick- } \\
\text { stoff }\end{array}$ & $\begin{array}{c}\text { Wasser- } \\
\text { stoff }\end{array}$ & $\begin{array}{c}\text { Kohlen- } \\
\text { oxyd }\end{array}$ & & ohargo & & $\begin{array}{l}\text { Rein- } \\
\text { argon }\end{array}$ & Mittel \\
\hline \begin{tabular}{c|}
1465 \\
1570 \\
1680 \\
1800 \\
1900 \\
2000 \\
2100 \\
2200 \\
2300 \\
2400 \\
2500 \\
2550 \\
2600 \\
2650 \\
nichtmehr \\
gemessen
\end{tabular} & $\begin{array}{r}\mathbf{5 , 0 5} \\
\mathbf{5 , 3 2} \\
\mathbf{5 , 6 6} \\
\mathbf{6 , 0 1} \\
\mathbf{6 , 3 1} \\
\mathbf{6 , 9 3} \\
\mathbf{7 , 3 0} \\
\mathbf{7 , 7 5} \\
\mathbf{8 , 3 3} \\
\mathbf{8 , 8 5} \\
\mathbf{9 , 1 0} \\
11,01\end{array}$ & $\begin{array}{l}\mathbf{5 , 0 4} \\
\mathbf{5 , 2 9} \\
\mathbf{5 , 6 3} \\
\mathbf{6 , 0 7} \\
\mathbf{6 , 3 3} \\
\mathbf{6 , 7 8} \\
\mathbf{7 , 4 4} \\
\mathbf{7 , 7 4} \\
\mathbf{8 , 3 3}\end{array}$ & $\begin{array}{l}\mathbf{5 , 1 1} \\
\mathbf{5 , 4 1} \\
\mathbf{5 , 5 9} \\
\mathbf{6 , 1 0} \\
\mathbf{6 , 3 9} \\
\mathbf{6 , 7 7} \\
\mathbf{7 , 4 7} \\
\mathbf{7 , 9 1} \\
\mathbf{8 , 2 7} \\
\mathbf{8 , 9 0}\end{array}$ & $\begin{array}{r}5,58 \\
6,04 \\
6,33 \\
6,65 \\
7,27 \\
7,81 \\
8,29 \\
8,88 \\
9,29 \\
10,54 \\
11,29 \\
\\
12,36\end{array}$ & $\begin{array}{r}\mathbf{5 , 6 2} \\
\mathbf{6 , 0 5} \\
\mathbf{6 , 3 2} \\
\mathbf{6 , 6 5} \\
\mathbf{7 , 2 1} \\
\mathbf{7 , 7 7} \\
\mathbf{8 , 2 7} \\
\mathbf{9 , 0 8} \\
\mathbf{1 0 , 0 1}\end{array}$ & $\begin{array}{r}\mathbf{6 , 0 9} \\
\mathbf{6 , 3 3} \\
\\
\mathbf{7 , 1 9} \\
\mathbf{7 , 6 5} \\
\mathbf{8 , 3 5}\end{array}$ & $\begin{array}{r}\mathbf{5 , 6 7} \\
\mathbf{6 , 0 6} \\
\mathbf{6 , 3 8} \\
\mathbf{6 , 7 3} \\
\mathbf{7 , 2 1} \\
\mathbf{7 , 6 7} \\
\mathbf{8 , 2 7} \\
\mathbf{8 , 7 8} \\
\mathbf{9 , 6 9} \\
\mathbf{1 0 , 4 0}\end{array}$ & $\begin{array}{r}\mathbf{5 , 0 7} \\
\mathbf{5 , 3 4} \\
\mathbf{5 , 6 3} \\
\mathbf{6 , 0 6} \\
\mathbf{6 , 3 4} \\
\mathbf{6 , 7 5} \\
\mathbf{7 , 3 0} \\
\mathbf{7 , 7 6} \\
\mathbf{8 , 3 0} \\
\mathbf{8 , 9 0} \\
\mathbf{9 , 5 2} \\
\mathbf{1 0 , 4 7} \\
11,15 \\
11,43 \\
12,26\end{array}$ \\
\hline
\end{tabular}

Die Resultate der auf diese Weise ausgeführten Versuche sind in Tabelle 2 zusammengestellt.

In der ersten Spalte findet sich die Temperatur, bei der die Schmelzen ans dem Ofen herausgenommen wurden, in Graden Celsius. Die darauffolgenden Spalten enthalten den Gesamtkohlenstoffgehalt der erkalteten Schmelzen, wobei die Überschrift der betreffenden Spalte angibt, welches Gas als schützende Atmosphäre Verwendung 
fand. Die letzte Spalte gibt die Mittelwerte aus den Ergebnissen aller bei gleicher Temperatur ausgeführten Versuche. Die Ergebnisse der in den verschiedenen Gasen ausgeführten Versuche unterscheiden sich bis $1900^{\circ} \mathrm{C}$ um höchstens $0,12 \%$, bis $2400^{\circ} \mathrm{C}$ um höchstens $0,3 \%$ und stimmen demnach ausgezeichnet miteinander überein. Die für $2500^{\circ} \mathrm{C}$ erhaltenen Werte unterscheiden sich allerdings urn nahezu $1 \%$ voneinander. Man erkennt hier den ungünstigen Ein-

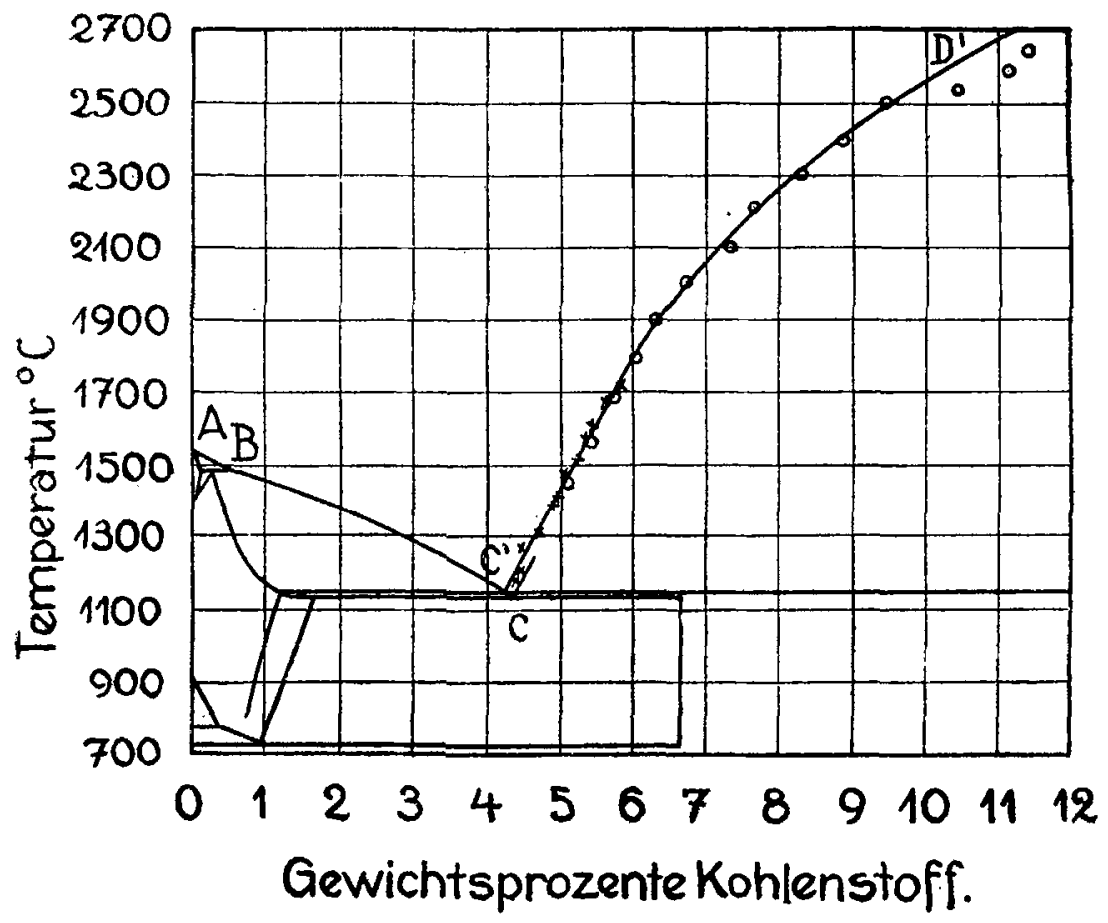

Fig. 5.

fluß, den die Rauchentwicklung auf die Genauigkeit der Temperaturmessung ausübt. Da diese mit wachsender Temperatur immer stärker wird, so können die für oberhalb $2500^{\circ} \mathrm{C}$ erhaltenen Werte nur als angenäherte gelten. Für die in der letzten Reiho von Tabelle 2 mitgeteilten Versuche, bei denen die Schmelzen über 12\% Kohlenstoff aufgenommon hatten, konnte der Rauchentwicklung wegen keine Temperaturmessung mehr ausgeführt werden. Sie lassen jedoch erkennen, daß auch bei Temperaturen über $2600^{\circ} \mathrm{C}$ der Kohlenstoffgehalt mit wachsender Temperatur noch zunimmt. Die Mittelwerte in Tabelle 2 sind in Fig. 5 durch kleine Kreise wiedergegeben. Die 
in der gleichen Figur durch Kreuze bestimmten Punkte entsprechen den in Tabelle 1, Spalte 8, angegebenen Gesamtkohlenstoffgehalten. Man erkennt die gute Übereinstimmung zwischen den Ergebnissen der Versuche mit thermoelektrischer und optischer Temperaturbestimmung in dem zwischen $1465^{\circ}$ und $1710^{\circ} \mathrm{C}$ liegenden Temperaturbereich. Durch die Kreuze und Kreise läßt sich ungezwungen eine Kurve $C^{\prime} D^{\prime}$ hindurchlegen, wobei nur die Ergebnisse der oberhalb $2500^{\circ} \mathrm{C}$ ausgeführten Versuche etwas herausfallen. Die Kurve verläuft von $1152^{\circ}$ bis fast $1700^{\circ} \mathrm{C}$ linear und biegt dann zanächst schwach und mit steigender Temperatur allmählich stärker konkav zur Konzentrationsachse ab. Einen Umkehrpunkt besitzt sie nicht.

Tabelle 3 .

\begin{tabular}{c|c}
\hline Temperatur ${ }^{\circ} \mathrm{C}$ & $\begin{array}{c}\text { Löslichkeit des Graphits } \\
\text { in Gewichtsprozenten }\end{array}$ \\
\hline 1152 & $\mathbf{4 , 2 5}$ \\
1200 & $\mathbf{4 , 3 7}$ \\
1300 & $\mathbf{4 , 6 0}$ \\
1400 & $\mathbf{4 , 8 5}$ \\
1500 & $\mathbf{5 , 1 0}$ \\
1600 & 5,37 \\
1700 & 5,67 \\
1800 & 6,00 \\
1900 & 6,37 \\
2000 & 6,78 \\
2100 & 7,24 \\
2200 & 7,72 \\
2300 & 8,26 \\
2400 & 8,88 \\
2500 & $\mathbf{9 , 5 4}$
\end{tabular}

Da keine Knicke auf ihr auftreten, so besteht sie aus einem einzigen Aste und ist ihr Verlauf ein solcher, wie er für die Löslichkeitskurve einer einzigen Kristallart zu erwarten ist. Ihre Gestalt läBt also einen Stabilitätswechsel der mit der Schmelze koexistierenden Kristallart äußerst unwahrscheinlich erscheinen. $\mathrm{DaB}$ ein solcher zwischen $1720^{\circ} \mathrm{C}$ und der eutektischen Erstarrungstemperatur thermisch nicht nachweisbar ist, wurde schon oben erwähnt. Die Kurve stellt also in ihrem ganzen Verlaufe die Löslichkeit des Graphits in geschmolzenem Eisen dar.

In Tabelle 8 ist die Löslichkeit des Graphits in flüssigem Eisen für die eutektische Temperatur $1152^{\circ}$, sodann für $1200^{\circ}$ und von $100 \mathrm{zu} 100^{\circ}$ fortschreitend bis $2500^{\circ} \mathrm{C}$ gegeben. 


\section{Die Zähigkeit der geschmolzenen und das Gefüge der orstarrten Eisen-Kohlenstofflegierungen.}

Erhitzt man das schwedische Holzkohlenroheisen mit 3,8\% Kohlenstoff ohne Graphitzusatz in einem Probierrohre aus Magnesia auf etwa $2400^{\circ} \mathrm{C}$, so bleibt es vollkommen dünnflüssig. Wird es dagegen im Probierrohre aus Kohle geschmolzen, sc läßt sich beim Ausgießen in die Kokille schon bei etwa $1800^{\circ} \mathrm{C}$ eine merkbare Zunahme der Zähflüssigkeit erkennen, indem die Menge der in denengen Raum der Kokille einfließenden Schmelze deutlich abnimmt. Diese Zähflüssigkeit, welche $\mathrm{H}_{\text {AnEmanN }}{ }^{1}$ ) schon bei $1700^{\circ} \mathrm{C}$ beobachtete, wächst mit steigender Temperatur und bewirkt, daB eine auf $2300^{\circ} \mathrm{C}$ erhitzte Schmelze auch durch StoB nicht mehr zum Ausfließen gebracht werden kann. Erhitzt man noch höher, so wird die Schmelze schließlich ganz teigartig; auf $2500^{\circ} \mathrm{C}$ und darüber erhitzte Schmelzen, welche im Probierrohre erkaltet waren, lieBen die Stelle erkennen, an der der Rührstab vor der Herausnahme aus dem Röhrchen gesessen hatte. Eine Wiederabnahme der Zühflüssigkeit mit steigender Temperatur konnte nicht beobachtet werden.

Wurde eine Schmelze im Probierrchr aus Kohle bis $2800^{\circ} \mathrm{C}$ erhitzt und dann langsam bis auf $1600^{\circ} \mathrm{C}$ abkühlen gelassen, so war sie so dickflüssig geblieben, daß sie nicht aus dem SchmelzgefäBe ausfloß. Das gleiche Resultat ergab sich, wenn die Schmelze nach der Erhitzung erstarren gelassen und dann wieder auf $1600^{\circ} \mathrm{C}$ erhitzt wurde. Man mußte vielmehr, um eine auf $2300^{\circ} \mathrm{C}$ erhitzt gewesene Schmelze wieder dünnflüssig zu erhalten, sie vor dem Wiederschmelzen auf gewöhnliche Temperatur abkühlen lassen.

Aus diesen Beobachtungen geht hervor, $\mathrm{da} B$ der gelöste Kohlenstoff die Diekflüssigkeit der Schmelze hervorruft. Die Temperatur wirkt nur insoweit, als sie die Löslichkeit des Kohlenstoffs im Eisen vergrößert. Die Beobachtung, daß hocherhitzte Eisen-Koblenstofflegierungen mit sinkender Temperatur nicht sofort wieder dünnflüssig werden, sondern daß es dazu nötig ist, sie vor dem emeuten Schmelzen auf gewöhnliche Temperatur erkalten zu lassen, ist wohl so zu erklären, da $B$ der beim Abkühlen der Schmelze in reichlicher Menge ausgeschiedene Graphit ein dichtes Netzwerk bildet, welches die Flüssigkeit am Ausfließen hindert. Wird dieses Netzwerk durch die Abkühlung des erstarrten Regulus auf Zimmertemperatur und vielleicht auch durch die nachfolgende Wiedererhitzung zerstört, so

1) Han gManN, Z. anorg. Chem. 84 (1914), l. 
kann die Schmelze ungehindert ausflieBen. Die Frage, ob mit der Aufnahme des Kohlenstoffs eine molekulare Veränderung verknüpft ist, wird durch die oben mitgeteilten Versuche nicht entschieden. Auf eine Ausscheidung von Graphit kann das Zähewerden beim Erhitzen auf keinen Fạll zurückgeführt werden, da dessen Löslichkeit mit steigender Temperatur zunimmt.

Die Schliffe wurden sowobl im ungeätzten Zustande, als auch nach vorhergehender Ätzung mit alkoholischer Pikrinsäure untersucht. Die bis etwa $1500^{\circ} \mathrm{C}$ erhitzten und dann in der Kokille abgeschreekten Proben enthielten primär in Form von Nadeln oder Blöcken ausgeschiedenen Zementit, umgeben von Zementiteutektikum. Wurden sie auf höhere Temperatur erhitzt, so trat daneben Graphit in mit steigender Temperatur zunehmender Menge auf, während gleichzeitig die Menge des primär abgesohiedenen Zementits abnahm. Daß dies eine Folge der für die hochgekohlten Schmelzen ungenügenden Abschreckungsgeschwindigkeit ist, ergibt sich, auch daraus, daß in diesen Fällen der primär ausgeschiedene Zementit hauptsächlich am Rande der Gußplatten, wo die Abkühlung am schnellsten erfolgt war, auftrat. Die Dicke der ausgeschiedenen Graphitlamellen wuchs mit der Höhe der Erhitzungstemperatur. An einzelnen Stellen der Schliffe waren die Graphitlamellen anscheinend durch Schleifen und Polieren entfernt worden und an ihrer Stelle Hohlräume vorhanden. Zuweilen wurden auch Tannenbaumkristalle in geringer Menge beobachtet. HANEMANN (1. c.) hat nachgewiesen, da $B$ es sich hier um Austenit handelt und sein Auftreten damit erklärt, daß eine Schmelze, welche durch Graphitausscheidung auf die Konzentration des Graphiteutektikums gebracht ist, Mischkristalle ausscheiden muß, um als Zementiteutektikum zu erstarren. Außer Graphit, Zementit und Austenit bzw. dessen Zerfallsprodukten konnten keine Kristallarten beobachtet werden. Die Beschaffenheit der Struktur spricht also nicht für das Auftreten weiterer, von dem Zementit verschiedener Karbide.

\section{Zusammenfassung.}

1. Es wurde die Löslichkeit des Graphits in geschmolzenem Eisen in Abhängigkeit von der Temperatur vc.n $1152-2500^{\circ} \mathrm{C}$ bestimmt (Tabelle 3). Oberhalb $2500^{\circ} \mathrm{C}$ bis etwa $2700^{\circ} \mathrm{C}$ konnten nur angenäherte Bestimmungen ausgeführt werden, welche ergaben, da $B$ auch noch in diesem Temperaturbereich die Löslichkeit des Graphits mit wachsender Temperatur zunimmt. 
112 R. Ruer u. J. Biren. Über d. Lössliehlieit d. Graphits in geschmolz. Eisen.

2. Die Löslichkeitskurve des Graphits in geschmolzenem Eisen verläuft von $1152^{\circ} \mathrm{C}$ bis fast $1700^{\circ} \mathrm{C}$ linear und biegt dann zunächst schwach und mit steigender Temperatur allmählich stärker konkav zur Konzentrationsachse ab.

3. In den Schliffen der erstarrten Proben konnte außer Graphit, Zementit und Austenit bzw. dessen Zerfallsprodukten keine Kristallart beobachtet werden.

4. Die Konzentration des Graphiteutektikums, dessen Erstarrungstemperatur $1152^{\circ} \mathrm{C}$ ist, liegt bei $4,25 \%$ Kohlenstoff, die des Zementiteutektikums, dessen Erstarrungstemperatur $1145^{\circ} \mathrm{C}$ ist, bei $4,30 \%$ Kohlenstoff.

5. Die mit steigender Temperatur zunehmende Dickflüssigkeit des in Berührung mit Kohlenstoff geschmolzenen Eisens rührt von dem gelösten Kohlenstoff her. Die Temperatur wirkt nur insoweit, als sie die Löslichkeit des Kohlenstoffs im Eisen vergrößert.

Aachen, Institut für theoretische Hüttenkunde und physikalischo Chemie der technischen Hochschule.

Bei der Redaktion eingegangen an 15. Juni 1920. 